\title{
Clinical verification of Lou type warfarin pharmacokinetic dosing algorithms equation
}

\author{
JIANGANG JIANG ${ }^{1}$, NINGNING JI ${ }^{2}$, JINGLIANG LAN ${ }^{1}$, XIAOPING GE ${ }^{3}$ and XIAOMA DU ${ }^{1}$ \\ ${ }^{1}$ Department of Cardiology, Jinhua Hospital of TCM Affiliated to Zhejiang University of Traditional Chinese Medicine, \\ Jinhua, Zhejiang 321000; ${ }^{2}$ Department of Cardiology, Yiwu Central Hospital, Yiwu, Zhejiang 322000; \\ ${ }^{3}$ Department of Geriatrics, Zhejiang Jinhua Guangfu Hospital, Jinhua, Zhejiang 321000, P.R. China
}

Received December 23, 2016; Accepted December 22, 2017

DOI: $10.3892 / \mathrm{mmr} .2018 .8562$

\begin{abstract}
Warfarin is the most commonly used oral anti-coagulant in clinic practice. However, it is difficult to recommend the correct dosage due to its narrow therapeutic window. The aim of the present study was to verify the clinical value of the Lou type equation, using pharmacogenetics-based warfarin dosing algorithms to appropriately predict the actual maintenance dose. A total of 87 Chinese Han patients who required treatment with warfarin were enrolled and randomly divided into the experimental and control groups. In the experimental group, the first 3 doses of warfarin were calculated according to the Lou type equation. While in the control group, these 3 treatments were performed following the doctors' recommendations. Then the dose of warfarin was gradually adjusted to the stable dose according to the changes in the international standardized ratio. At the end of the 50 day experimental period, there were a greater number of patients in the experimental group who exhibited a stable blood concentration of warfarin than those in the control group (83.35 and $64.4 \%$, respectively). In addition, the mean and median times for patients to obtain a stable dose in the experimental group were significantly shorter than those in the control group (mean, 18.2 \pm 1.7 and 27.3 \pm 2.0 days; and median, 11.7 \pm 1.1 and $20.5 \pm 1.8$ days, respectively). The adverse reaction rate of the experimental group (9.5\%) was markedly lower than that of the control group (26.7\%). The occurrence of adverse reactions in the experimental group was also significantly later when compared with the control group (43.9 \pm 1.6 and 38.6 \pm 1.5 days, respectively). Furthermore, there was no significant difference between the average predicted dose $(3.4 \pm 1.1 \mathrm{mg} /$ day $)$ and the average actual dose $(3.5 \pm 1.4 \mathrm{mg} / \mathrm{day} ; \mathrm{P}=0.313)$. In conclusion,
\end{abstract}

Correspondence to: Dr Jiangang Jiang, Department of Cardiology, Jinhua Hospital of TCM Affiliated to Zhejiang University of Traditional Chinese Medicine, 439 Shuangxi West Road, Wucheng, Jinhua, Zhejiang 321000, P.R. China

E-mail: jjg2009768@163.com

Key words: warfarin, Lou type equation, dose prediction algorithm, pharmacogenetics, gene polymorphism using the Lou type warfarin pharmacokinetic dosing algorithm equation to administer warfarin markedly shortened the adjustment time of warfarin to reach a stable dose and reduced the adverse reactions rate, thus supporting clinical feasibility.

\section{Introduction}

Warfarin is the most commonly used oral anti-coagulant in clinical practice and is widely used in the prevention and treatment of various types of deep venous thrombosis, pulmonary embolism, prosthetic heart valves and atrial fibrillation. Warfarin is characterized by its narrow therapeutic window, which means that a small change in concentration can cause serious side effects $(1,2)$. Due to differences in the metabolism of warfarin in individuals, inadequate or excessive use of warfarin can lead to thrombus or hemorrhage $(1,2)$. Genetic and non-genetic factors, including age, sex, race, weight and drug interactions can account for the better patient responses to specific doses (2-5). So the genetic polymorphisms contribute to the individual differences in warfarin dose response (3-5). A number of clinical research studies have revealed that a predictive equation based on pharmacogenomics is reliable for determining the dosage that is affected by individuals' genetic characteristics (6-11). Genetic polymorphisms of cytochrome $\mathrm{P} 450$ family 2 subfamily $\mathrm{C}$ member 9 (CYP2C9), vitamin $\mathrm{K}$ epoxide reductase complex subunit 1 (VKORC1) and cytochrome P450 family 4 subfamily $\mathrm{F}$ member 2 (CYP4F2) may result in $30-50 \%$ of individual warfarin dose variability (12-14). Lane et al (15) reported that CYP2C19 and $C Y P 3 A 4$ genotypes had a profound effect on R-warfarin clearance. Rieder et al (16) found that the $\gamma$-glutamyl carboxylase $(G G C X)-12970$ SNP was associated with the warfarin maintenance dose. Chung et al (17) confirmed that polymorphisms of microsomal epoxide hydrolase 1 (EPHX1) and VKORC1-like 1 (VKORC1L1) could contribute to the warfarin dose variability. A number of studies and clinical trials regarding the pharmacogenomics of warfarin have been conducted worldwide, and they have yielded a number of different drug equations $(4,5,10,18,19)$. However, the value of each equation in the clinical application between different races in different regions is quite different (3-5,7,13). A study published by Lou et al (5) in May 2014 identified the stable dose of warfarin by evaluating Han Chinese patients and the 
results were of particular interest. The Lou type warfarin pharmacokinetic dosing algorithm equation was based on the genetic polymorphisms of CYP2C9, VKORC1, and CYP4F2 and other non-genetic variables. In the present study, the Lou type warfarin pharmacokinetic dosing algorithm equation was applied to verify the efficacy of warfarin in clinical treatments via a randomized, controlled prospective study of Han Chinese patients in Zhejiang, which supported the implementation of medicine stemming from clinical studies.

\section{Materials and methods}

Patients. The present study was approved by the Ethics Committee of the Jinhua Hospital of TCM Affiliated to Zhejiang University of Traditional Chinese Medicine (Zhejiang, China). Patients ( $\mathrm{n}=87$; Table I) who were admitted to Jinhua Hospital of TCM Affiliated to Zhejiang University of Traditional Chinese Medicine (Zhejiang, China), Yiwu Central Hospital (Zhejiang, China), and Zhejiang Jinhua Guangfu Hospital (Zhejiang, China) and Rehabilitation Hospital of Yiwu (Zhejiang, China) and required warfarin treatment were recruited to the present study between June 2014 to 2016. Written informed consent was obtained from all participants.

Inclusion and exclusion criteria. The inclusion criteria were as follows: i) Patients were $>18$ years and were able to behave independently; ii) patients with sufficient medical data available; and iii) patients consented to a 50 day experimental period following treatment. The exclusion criteria were as follows: i) Patients with no serious bleeding within the last 6 months; ii) patients who rejected long-term anti-coagulant therapy; and iii) patients were unable to complete the study.

Lou type warfarin pharmacokinetic dosing algorithm equation. The present study selected the Lou type warfarin pharmacokinetic dosing algorithm equation following comparisons between the equations from multiple studies $(1,5,7,18)$. The warfarin stable dosage equation was as follows: Daily dose of warfarin $(\mathrm{mg})=1.087+2.226 \mathrm{x}$ VKORC1(1639AG) $)^{\#}+3.844 \times$ VKORC1(1639GG) $)^{\$}-1.284$ x CYP2C9 $\left({ }^{*} 1 /^{*} 3\right)^{\&}-2.182$ x CYP2C9( $\left.{ }^{*} 3 /^{*} 3\right)^{\alpha}+0.221$ x CYP4F2(CT) $)^{\beta}+0.336 \times$ CYP4F2(TT) $)^{\gamma}-0.018 \times$ age (years) $+0.015 \times$ weight $(\mathrm{kg})+0.013 \times$ height $(\mathrm{cm})-0.777 \times$ Amiodarone $^{\lambda}-0.379 \mathrm{x}$ digoxin ${ }^{\sigma}$. Where the following have been applied: "VKORC1(1639AA) = 0, VKORC1 $(1639 A G)=1$; ${ }^{\$} \mathrm{VKORC} 1(1639 \mathrm{AA})=0, \operatorname{VKORC} 1(1639 \mathrm{GG})=1$; ${ }^{\&} \mathrm{CYP} 2 \mathrm{C} 9\left({ }^{*} 1 /{ }^{*} 1\right)=0, \mathrm{CYP} 2 \mathrm{C} 9\left({ }^{*} 1 /{ }^{*} 3\right)=1 ;{ }^{\alpha} \mathrm{CYP} 2 \mathrm{C} 9\left({ }^{*} 1 /{ }^{*} 1\right)=0$, $\mathrm{CYP} 2 \mathrm{C} 9\left({ }^{*} 3 /{ }^{*} 3\right)=1 ;{ }^{\beta} \mathrm{CYP} 4 \mathrm{~F} 2(\mathrm{CC})=0, \mathrm{CYP} 4 \mathrm{~F} 2(\mathrm{CT})=1$; ${ }^{\gamma} \mathrm{CYP} 4 \mathrm{~F} 2(\mathrm{CC})=0, \mathrm{CYP} 4 \mathrm{~F} 2(\mathrm{TT})=1 ;{ }^{\lambda}$ used amiodarone $=1$, did not use amiodarone $=0 ;{ }^{\sigma}$ used digoxin $=1$, did not use digoxin $=0$.

Information collection. According to Table I, 87 patients prescribed with anti-clotting warfarin were randomly divided into the experimental and control groups. Clinical data and blood samples were then collected. The personal data of each participant, including age, sex, height, weight, medical history, hemorrhage history and medication history were recorded. A total of $5 \mathrm{ml}$ blood was obtained from each patient for the present study.
Polymerase chain reaction-restriction fragment length polymorphism (PCR-RFLP) and sequencing. The polymorphism of CYP2C9, VKORC1, and CYP4F2 were detected by PCR-RFLP and DNA sequencing. Peripheral white blood cell genomic DNA were extracted from blood samples using the PAXgene Blood DNA kit (Qiagen, GmbH, Hilden, Germany) (the Japanese supplier being Cosmo Bio Co., Ltd., Tokyo, Japan), according to the manufacturer's instructions. According to the method described previously (20,21), CYP2C9*3 (1075A/C, rs1057910), VKORC1 (1639G/A, rs9923231) and CYP4F2 V433M (rs2108622) were amplified by PCR. Then, the PCR products were digested for $1.5 \mathrm{~h}$ at $37^{\circ} \mathrm{C}$ with a restriction endonuclease, and analyzed by $2 \%$ agarose gel electrophoresis. The DNA sequences of all samples were visualized by ethidium bromide under ultraviolet light using the Molecular Imager Chemi Doc XRS+ System (Bio-Rad Laboratories, Inc., Hercules, CA, USA) and analyzed with Quantity One 1D Analysis software (version 4.6.6; Bio-Rad Laboratories, Inc.). Then, all samples were analyzed by DNA sequencing in Sangon Biotech (Shanghai) Co., Ltd. (Shanghai, China).

Study strategy. To evaluate the equation in a single-blind manner, the patients were divided into 2 groups without any knowledge of which group they were assigned to. The polymorphism of the 3 genes was determined and the predicted dose of warfarin was calculated according to the Lou type equation, as aforementioned. For the first 3 administrations, all patients in the control group were treated according to the dosages prescribed by an experienced doctor, while all participants in the experimental group were treated using the dosages calculated by the Lou type equation. Then, the dose of warfarin administrated to all patients was gradually adjusted to a stable dose according to the changes in the international standardized ratio (INR). The stable dose means that after the same patient received the same dose, the two consecutive INR values were between 2.0-3.0, and the interval time was $>7$ days. Following the 50 day experimental period following the first administration, the stable dose, the time to achieve a stable dose and adverse reactions were recorded. In addition, the physician in charge was informed of the study's results and appropriate measures were applied if adverse reactions, including INR $>3.5$, bleeding, or new thrombosis occurred.

Statistical analysis. All analyses were performed using Stata 12.0 software (StataCorp LP, College Station, TX, USA). Quantitative data are presented as the mean \pm standard error of the mean. Measurement data were analyzed using a paired t-test and the enumeration data were analyzed with an $\chi^{2}$ test. $\mathrm{P}<0.05$ was considered to indicate a statistically significant difference.

\section{Results}

Sequencing analysis. PCR-RFLP was used to detect the genotypes of CYP2C9*3 (1075A/C, rs1057910), VKORC1 (1639G/A, rs9923231) and CYP4F2 V433M (rs2108622). The amplification products of CYP2C9, VKORC1 and CYP4F2 (V433M) were 200, 423 and 491 bp, respectively. Furthermore, 
Table I. Comparison of clinical parameters and genotypes between the experimental and control groups.

\begin{tabular}{|c|c|c|c|}
\hline Clinical parameters & Experimental group $(n=42)$ & Control group $(n=45)$ & P-value \\
\hline Age (years) & $62.5 \pm 12.8$ & $61.4 \pm 13.2$ & 0.628 \\
\hline Sex (female/male) & $17 / 25$ & $17 / 28$ & 0.797 \\
\hline Height (cm) & $160.2 \pm 8.2$ & $159.5 \pm 8.6$ & 0.649 \\
\hline Weight (kg) & $65.2 \pm 10.9$ & $66.8 \pm 11.3$ & 0.458 \\
\hline Anticoagulation indications & & & 0.876 \\
\hline Atrial fibrillation (n) & 29 & 32 & \\
\hline Venous thrombosis (n) & 5 & 7 & \\
\hline Pulmonary embolism (n) & 3 & 2 & \\
\hline Valve replacement (n) & 5 & 4 & \\
\hline Combined use of drugs & & & 0.905 \\
\hline Amiodarone (n) & 2 & 2 & \\
\hline Digoxin (n) & 8 & 7 & \\
\hline CYP2C9 & & & 0.808 \\
\hline$* 1 / * 1(\mathrm{n})$ & 38 & 40 & \\
\hline$* 1 / * 3(n)$ & 4 & 5 & \\
\hline$* 3 / * 3(\mathrm{n})$ & 0 & 0 & \\
\hline VKORC1 & & & 0.563 \\
\hline $\mathrm{AA}(\mathrm{n})$ & 35 & 35 & \\
\hline $\mathrm{AG}(\mathrm{n})$ & 7 & 9 & \\
\hline GG (n) & 0 & 1 & \\
\hline CYP4F2 & & & 0.849 \\
\hline $\mathrm{CC}(\mathrm{n})$ & 22 & 22 & \\
\hline $\mathrm{CT}(\mathrm{n})$ & 16 & 17 & \\
\hline TT (n) & 4 & 6 & \\
\hline
\end{tabular}

CYP2C9, cytochrome P450 family 2 subfamily C member 9; VKORC1, vitamin K epoxide reductase complex subunit 1; CYP4F2, cytochrome P450 family 4 subfamily F member 2.

the mutated amplification product of CYP2C9*3 $(1075 \mathrm{~A} / \mathrm{C})$ was digested by KpnI into 2 fragments of 180 and 20 bp; the mutated amplification product of VKORC1 (1639G/A) can be digested by MspI into 2 fragments of 207 and 216 bp; and the mutated amplification product of CYP4F2 V433M can be digested by PvuII into 2 fragments of 319 and $178 \mathrm{bp}$. According to the results of enzyme digestion, there were 3 genotypes of each gene: Mutant homozygote, heterozygote and wild-type (Fig. 1; Table I). All samples were analyzed by DNA sequencing and the results were consistent with those of PCR-RFLP.

Comparison of the clinical parameters. The 87 patients were divided into 2 groups, with 42 participants in the experimental group and 45 participants in the control group. No significant differences were identified in the distribution of the CYP2C9*3, VKORC1 (1639G/A) and CYP4F2 V433M genotypes, age, sex, height, weight and history of disease (Table I) between the 2 groups.

Comparisons of the number of patients exhibited a stable dose, the time for patients to obtain a stable dose and the adverse reactions. According to the obtained statistics, there was a significant difference in the number of patients reaching a stable dose between the 2 groups, with 35 patients (83.3\%) in the experimental group and 29 patients $(64.4 \%)$ in the control group $(\mathrm{P}=0.046)$. In addition, it took $18.2 \pm 1.7$ days for the participants in the experimental group to achieve a stable dose, which was less than that of the control group (27.3 \pm 2.0 days). The median time to achieve a stable dose was $11.7 \pm 1.1$ days for the experimental group and $20.5 \pm 1.8$ days for the control group, which indicated a statistically significant difference between the two groups $(\mathrm{P}<0.001)$. Furthermore, the incidence of adverse reactions in the experimental group was $9.5 \%$ (4 cases), which was significantly lower than that of the control group $(26.7 \%$; 12 cases; $\mathrm{P}=0.039)$. In addition, the average time of developing adverse reactions in the experimental group $(43.9 \pm 1.6$ days) was significantly longer than that of the control groups $(38.6 \pm 1.5$ days; $\mathrm{P}=0.046)$.

Comparisons between the predictive dose and actual dose. At the end of the study, 64 participants received the stable dose aggregately. The average dose of $3.4 \pm 1.1 \mathrm{mg} /$ day, which was predicted using the Lou type equation, was lower than the actual average dose of $3.5 \pm 1.4 \mathrm{mg} /$ day; however, no significant difference was observed between the predicted dose and the actual dose $(\mathrm{P}=0.313)$. 
A

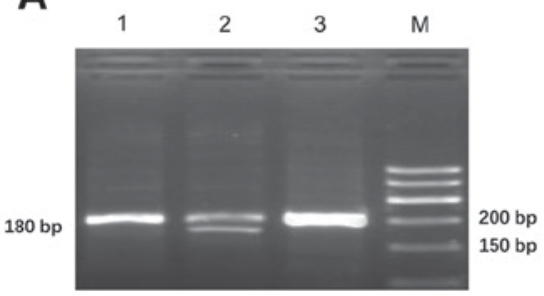

B

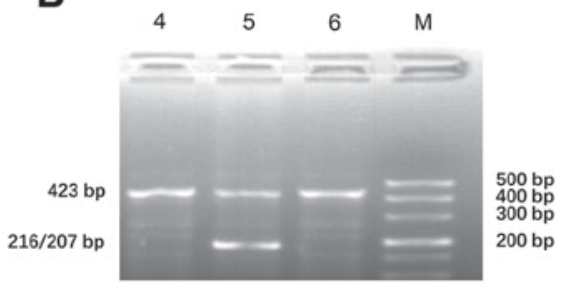

C

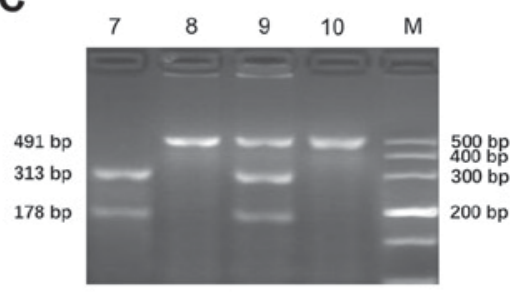

Figure 1. Genotype analysis of (A) CYP2C9*3 (1075A/C, rs1057910), (B) VKORC1 (1639G/A, rs9923231) and (C) CYP4F2 V433M (rs2108622). Lane M, DNA marker; lane 1, wild-type of CYP2C9 ("1/*1); lane 2, heterozygote of CYP2C9 ("1/*3); lane 3, amplification product of CYP2C9 by PCR; lane 4, wild-type of VKORC1(1639A/A); lane 5, heterozygote of VKORC1(1639A/G); lane 6, amplification product of VKORC1 by PCR; lane 7, mutant homozygote of CYP4F2 V433M (TT); lane 8, wild-type of CYP4F2 V433M (CC); lane 9, heterozygote of CYP4F2 V433M (CT); lane 10, amplification product of CYP4F2 V433M by PCR; PCR, polymerase chain reaction; CYP2C9, cytochrome P450 family 2 subfamily C member 9; VKORC1, vitamin K epoxide reductase complex subunit 1; CYP4F2, cytochrome P450 family 4 subfamily F member 2.

\section{Discussion}

Individualized treatment refers to the use of individual drugs and also the use of individualized drug dosages based on pharmacogenomics, which has become a popular method for treating cardiovascular illnesses (22). Warfarin is one of the preferred anti-coagulant drugs for the anti-coagulant therapy in patients with atrial fibrillation, deep vein thrombosis, pulmonary embolism, cerebral infarction, radiofrequency ablation, and multiple types of cardiomyopathy at present (23). Previous studies have demonstrated that non-genetic factors including sex, body weight, body surface area, age, number of increasing INR drugs, smoking habit, preoperative stroke history and hypertension were minor determinants of warfarin stable dosage $(6,19,24)$. Thus, the problem of how to determine the stable dosage and how to maintain the dose accurately and conveniently should be addressed.

Some previous studies focusing on pharmacogenomics have indicated that the single nucleotide polymorphism (SNP) of CYP2C9*3 and VKORC1(1639G/A) may affect the pharmacodynamics and pharmacokinetics of warfarin $(3,4,8-11,14,22$, $24,25)$. In addition, other previous studies have suggested that the SNP of CYP4F2 V433M may be a determinant of warfarin's pharmacodynamic and pharmacokinetic properties (21).

Although numerous equations for predicting a stable dose of warfarin have been certificated, their practical application value has differed $(4,5,7,19,22,26)$. A number of verification tests performed for these equations did not produce perfect results (27-29). However, the results were worse still in clinics, as many studies from China were only based on a single disease, including atrial fibrillation, pulmonary embolism or valve replacement; particularly in the cardiovascular departments of primary hospitals, where warfarin was often used for atrial fibrillation, and in larger hospitals, where warfarin was used for valve replacements $(30,31)$. In some cases, combining warfarin with digoxin treatment has produced better results in patients with rheumatic heart disease or chronic heart failure; however, patients who also had valve replacement may require the combination of warfarin with amiodarone (32-35). Therefore, the warfarin stable dose prediction equation derived by taking into account a variety of factors that affect the stability of warfarin dose may be the best option for patients and doctors. Following comparison with other equations, it was demonstrated that the Lou type equation used in the present study may have greater practical value in the Chinese Han population $(1,5,7,21,26-28)$.

In the present study, the value of the Lou type warfarin pharmacokinetic dosing algorithm equation was verified through a randomized controlled prospective study of Han Chinese patients in Zhejiang. The results indicated that the experimental group yielded a greater number of cases reaching a stable dose and took less time to achieve a stable dosage than the control group. Therefore, using the Lou type equation may significantly shorten the dosage adjusting time, facilitate an effective and stable drug concentration, reduce detection by INR and even decrease therapeutic costs.

Furthermore, the incidence of adverse reactions in the control group was $26.7 \%$ (12 cases), which was significantly higher than that of the $9.5 \%$ in the experimental group (4 cases). In addition, the experimental group took $43.9 \pm 1.6$ days to develop adverse reactions, while these reactions were observed in the control group following $38.6 \pm 1.5$ days. Thus, using the Lou type equation may reduce the incidence of side effects and delay the occurrence of adverse reactions, resulting in a safer clinical application of warfarin.

In the present study, 65 participants received the stable dose aggregately. The average dose of $3.5 \pm 1.1 \mathrm{mg} / \mathrm{day}$, which was predicted by the Lou type equation, was lower than the actual average dose of $3.5 \pm 1.4 \mathrm{mg} /$ day, however, no significant difference was found between the two groups. The results of the present study are indicative of the strong application value of the Lou type warfarin pharmacokinetic dosing algorithm equation for treating Han Chinese patients.

Although the present study yielded favorable results, it did have some limitations. For individuals, the predicted stable dose was an estimate that may have a large discrepancy with the actual value, likely leading to an unnecessary clinical risk. Therefore, the predicted stable dose should be considered as a reference in the process of adjusting dosages. Doctors or physicians should be more concerned with the monitored INR. In terms of estimating eating habits, drug use and health should not be underestimated. As the present study did not adjust dosages for factors including eating habits, socioeconomic status and patients' cognition regarding warfarin, a local large-scale study is required to yield more accurate data.

In conclusion, the application of Lou type warfarin pharmacokinetic dosing algorithm equation markedly shortened the adjustment time and reduced the occurrence of adverse 
reactions, which suggested that it may have great value in clinical drug application.

\section{Acknowledgements}

Not applicable.

\section{Funding}

No funding was received.

\section{Availability of data and materials}

The analyzed data sets generated during the study are available from the corresponding author on reasonable request.

\section{Authors' contributions}

JGJ designed the study, performed the experiments, analyzed the data and wrote the manuscript. NNJ, JLL, XPG and XMD collected the cases. All authors read and approved the final manuscript.

\section{Ethics approval and consent to participate}

The present study was approved by the Ethics Committee of the Jinhua Hospital of TCM Affiliated to Zhejiang University of Traditional Chinese Medicine (Jinhua, Zhejiang, China). Written informed consent was obtained from all participants.

\section{Consent for publication}

Written informed consent was obtained from all participants.

\section{Competing interests}

All authors declare that they have no competing interests.

\section{References}

1. Yu Z, Ding YL, Lu F, Miao LY, Shen ZY and Ye WX: Warfarin dosage adjustment strategy in Chinese population. Int J Clin Exp Med 8: 9904-9910, 2015.

2. Baglin TP, Keeling DM and Watson HG; British Committee for Standards in Haematology: Guidelines on oral anticoagulation (warfarin): Third edition-2005 update. Br J Haematol 132: 277-285, 2006

3. Park SM, Lee JK, Chun SI, Lee HI, Kwon SU, Kang DW and Kim JS: VKORC1 and CYP2C9 genotype variations in relation to warfarin dosing in Korean stroke patients. J Stroke 15: 115-121, 2013.

4. Krishna Kumar D, Shewade DG, Loriot MA, Beaune P, Balachander J, Sai Chandran BV and Adithan C: Effect of CYP2C9, VKORC1, CYP4F2 and GGCX genetic variants on Warfarin maintenance dose and explicating a new pharmacogenetic algorithm in South Indian population. Eur J Clin Pharmacol 70: 47-56, 2014.

5. Lou Y, Hua L, Han L, Li Y, Zhang X, Tang M, Yu H, Liu Z, Wang W, Xu J, et al: Establishment and preliminary validation of Warfarin maintenance dose algorithm in Chinese Han Population. Zhonghua Xin Xue Guan Bing Za Zhi 42: 384-388, 2014 (In Chinese).

6. Routledge PA, Davies DM, Bell SM, Cavanagh JS and Rawlins MD: Predicting patients' warfarin requirements. Lancet 2: 854-855, 1977.

7. Li X, Liu R, Luo ZY, Yan H, Huang WH, Yin JY, Mao XY, Chen XP, Liu ZQ, Zhou HH and Zhang W: Comparison of the predictive abilities of pharmacogenetics-based warfarin dosing algorithms using seven mathematical models in Chinese patients Pharmacogenomics 16: 583-590, 2015.
8. Aithal GP, Day CP, Kesteven PJ and Daly AK: Association of polymorphisms in the cytochrome P450 CYP2C9 with warfarin dose requirement and risk of bleeding complications. Lancet 353: 717-719, 1999.

9. Bodin L, Verstuyft C, Tregouet DA, Robert A, Dubert L, Funck-Brentano C, Jaillon P, Beaune P, Laurent-Puig P, Becquemont L and Loriot MA: Cytochrome P450 2C9 (CYP2C9) and vitamin $\mathrm{K}$ epoxide reductase (VKORC1) genotypes as determinants of acenocoumarol sensitivity. Blood 106: 135-140, 2005.

10. Sconce EA, Khan TI, Wynne HA, Avery P, Monkhouse L, King BP, Wood P, Kesteven P, Daly AK and Kamali F: The impact of CYP2C9 and VKORC1 genetic polymorphism and patient characteristics up-on warfarin dose requirements: Proposal for a new dosing regiment. Blood 106: 2329-2333, 2005.

11. Rost S, Fregin A, Ivaskevicius V, Conzelmann E, Hörtnagel K, Pelz HJ, Lappegard K, Seifried E, Scharrer I, Tuddenham EG, et al: Mutations in VKORC1 cause warfarin resistance and multiple coagulation factor deficiency type 2 . Nature 427: 537-541, 2004.

12. Zhou SF, Zhou ZW and Huang M: Polymorphisms of human cytochrome P450 2C9 and the functional relevance. Toxicology 278: 165-188, 2010.

13. Namazi S, Azarpira N, Hendijani F, Khorshid MB, Vessal G and Mehdipour AR: The impact of genetic polymorphisms and patient characteristics on warfarin dose requirements: A cross-sectional study in Iran. Clin Ther 32: 1050-1060, 2010.

14. Higashi MK, Veenstra DL, Kondo LM, Wittkowsky AK, Srinouanprachanh SL, Farin FM and Rettie AE: Association between CYP2C9 genetic variants and anticoagulation related outcomes during warfarin therapy. JAMA 287: 1690-1698, 2002.

15. Lane S, Al-Zubiedi S, Hatch E, Matthews I, Jorgensen AL, Deloukas P, Daly AK, Park BK, Aarons L, Ogungbenro K, et al: The population pharmacokinetics of R- and S-warfarin: Effect of genetic and clinical factors. Br J Clin Pharmacol 73: 66-76, 2012.

16. Rieder MJ, Reiner AP and Rettie AE: Gamma-glutamyl carboxylase (GGCX) tagSNPs have limited utility for predicting warfarin maintenance dose. J Thromb Haemost 5: 2227-2234, 2007.

17. Chung JE, Lee KE, Chang BC and Gwak HS: Polymorphisms of vitamin K-related genes (EPHX1 and VKORC1L1) and stable warfarin doses. Gene 641: 68-73, 2018.

18. Hamberg AK, Hellman J, Dahlberg J, Jonsson EN and Wadelius M: A Bayesian decision support tool for efficient dose individualization of warfarin in adults and children. BMC Med Inform Decis Mak 15: 7, 2015.

19. International Warfarin Pharmacogenetics Consortium, Klein TE, Altman RB, Eriksson N, Gage BF, Kimmel SE, Lee MT, Limdi NA, Page D, Roden DM, et al: Estimation of the warfarin dose with clinical and pharmacogenetic data. N Engl J Med 360: 753-764, 2009.

20. Cui GL, Ding H, Xu YJ, et al: High resolution melting curves (HRM) in the prevention of Warfarin initiation guide measurement genotyping and clinical application of various genotyping methods. Chin J Cardiol 40: 477-481, 2012.

21. Deng S, Zhu G, Liu F, Zhang H, Qin X, Li L and Zhiyi H: CYP4F2 gene V433M polymorphism is associated with ischemic stroke in the male Northern Chinese Han population. Prog Neuropsychopharmacol Biol Psychiatry 34: 664-668, 2010.

22. Fung E, Patsopoulos NA, Belknap SM, O'Rourke DJ, Robb JF, Anderson JL, Shworak NW and Moore JH: Effect of genetic variants, especially CYP2C9 and VKORC1, on the pharmacology of Warfarin. Semin Thromb Hemost 38: 893-904, 2012.

23. Hirsh J, Dalen JE, Anderson DR, Poller L, Bussey H, Ansell J, Deykin D and Brandt JT: Oral anticoagulants: Mechanism of action, clinical effectiveness, and optimal therapeutic range. Chest 114 (5 Suppl): 445S-469S, 1998

24. Shahin MH, Cavallari LH, Perera MA, Khalifa SI, Misher A, Langaee T, Patel S, Perry K, Meltzer DO, McLeod HL and Johnson JA: VKORC1 Asp36Tyr geographic distribution and its impact on warfarin dose requirements in Egyptians. Thromb Haemost 109: 1045-1050, 2013.

25. Mushiroda T, Ohnishi Y, Saito S, Takahashi A, Kikuchi Y, Saito S, Shimomura H, Wanibuchi Y, Suzuki T, Kamatani N and Nakamura Y: Association of VKORC1 and CYP2C9 polymorphisms with warfarin dose requirements in Japanese patients. J Hum Genet 51: 249-253, 2006.

26. Chen J, Shao L, Gong L, Luo F, Wang J, Shi Y, Tan Y, Chen Q, Zhang Y, Hui R and Wang Y: A pharmacogenetics-based Warfarin maintenance dosing algorithm from Northern Chinese patients. PLoS One 9: e105250, 2014. 
27. Zhuang WF, Chen YH, Luo RP, et al: Evaluation on the stable dose prediction accuracy of Warfarin anticoagulant therapy by pharmacogenetics among Shanghai patients. Lab Med 30: 697-702, 2015.

28. Yu LP, Song HT, Zeng ZY, Wang QM and Qiu HF: Validation and comparison of pharmacogenetics-based warfarin dosing algorithms in Han Chinese Patients. Zhonghua Xin Xue Guan Bing Za Zhi 40: 614-619, 2012 (In Chinese).

29. Roper N, Storer B, Bona R and Fang M: Validation and comparison of pharmacogenetics-based warfarin dosing algorithms for application of pharmacogenetic testing. J Mol Diagn 12: 283-291, 2010

30. Zhao S, Zhao H, Wang X, Gao C, Qin Y, Cai H, Chen B and Cao J: Factors influencing medication knowledge and beliefs on warfarin adherence among patients with atrial fibrillation in China. Patient Prefer Adherence 11: 213-220, 2017.

31. Han ZH, Ren XJ and Wang Y: Anticoagulation management of patients with long-term warfarin therapy after valve replacement during the perioperative period of pacemaker implantation. Int J Clin Exp Med 6: 594-598, 2013.
32. Hudson M, Richard H and Pilote L: Differences in outcomes of patients with congestive heart failure prescribed celecoxib, rofecoxib, or non-steroidal anti-inflammatory drugs: Population based study. BMJ 330: 1370, 2005.

33. Cleland JG, Findlay I, Jafri S, Sutton G, Falk R, Bulpitt C, Prentice C, Ford I, Trainer A and Poole-Wilson PA: The Warfarin/Aspirin Study in Heart failure (WASH): A randomized trial comparing antithrombotic strategies for patients with heart failure. Am Heart J 148: 157-164, 2004.

34. Lu Y, Won KA, Nelson BJ, Qi D, Rausch DJ and Asinger RW: Characteristics of the amiodarone-warfarin interaction during long-term follow-up. Am J Health Syst Pharm 65: 947-952, 2008.

35. Zhong SL, Liu Y, Yu XY, Xu D, Tan HH, Lin QX, Yang M, Lao HY and Lin SG: The influence of genetic polymorphisms and interacting drugs on initial response to warfarin in Chinese patients with heart valve replacement. Eur J Clin Pharmacol 67: 581-590, 2011. 